\title{
Customer Feedback Analyzer
}

\section{Sanya Taneja, Kartikeya Jha, Nakul Lakhotia, Vedanta Kapoor, Swarnalatha P.}

\begin{abstract}
Product reviews always act as a great source of information for a company. These reviews record the customer's feedback on the product and services that the company provides. The problem that we face is that the number of reviews in these kinds of portals are in thousands for which manual analysis is time consuming and inefficient. So, we plan to make an automated system using machine learning which can do the job of analyzing large number of comments in seconds thereby increasing efficiency. These reviews which are posted online can be both positive and negative, categorizing them into broad categories like product defected, product size invalid, good fitting, excellent working etc., will ease the process for both consumers and sellers. This will help automate the process of Customer Resolution, as it takes a lot of time for an employee to manually sort each comment into various categories and then send it to the particular team for review. Additionally, trends can also be analyzed on these comments, such as which issue is most faced by consumers around a particular date/time. This project will efficiently extract the important topics of concern which the company should focus on and also change or improve in order to keep its customers happy and loyal.
\end{abstract}

Keywords: Customer Experience, Deep Neural Network, Natural language processing, Sentiment analysis

\section{INTRODUCTION}

Most of the companies have started to realize the importance of keeping their customers happy and understanding their needs. Many companies today resort to using technology for the purpose of analyzing the large volumes of customer feedback data like surveys, comments, suggestions and criticism. There are two things that companies want from this process - what customers hate or like about their product or service and if the comments are positive or negative. According to our study using machine learning algorithms for the task of sentiment analysis of text data proves to be more efficient than a human classifier. Sentiment Analysis is a special form of text categorization

Revised Manuscript Received on December 30, 2019.

* Correspondence Author

Sanya Taneja, School of Computer Science and Engineering, Vellore Institute of Technology, Vellore (Tamil Nadu) India. E-mail: sanyataneja32@gmail.com

Kartikeya Jha, School of Computer Science and Engineering, Vellore Institute of Technology, Vellore (Tamil Nadu) India. E-mail: kartikeyajha27@gmail.com

Nakul Lakhotia, School of Computer Science and Engineering, Vellore Institute of Technology, Vellore (Tamil Nadu) India. E-mail: nakullahotia@gmail.com

Vedanta Kapoor, School of Computer Science and Engineering, Vellore Institute of Technology, Vellore (Tamil Nadu) India. E-mail:vedantkapoor98@gmail.com

Swarnalatha P., School of Computer Science and Engineering, Vellore Institute of Technology, Vellore (Tamil Nadu) India. E-mail: hgswarna@gmail.com

(C) The Authors. Published by Blue Eyes Intelligence Engineering and Sciences Publication (BEIESP). This is an open access article under the CC BY-NC-ND license (http://creativecommons.org/licenses/by-nc-nd/4.0/)

which requires the machine learning algorithm to understand the context of sentence and accordingly classify the text as positive, negative or neutral. Python provides us with specific libraries which can be used for the text mining process.

Tamil Nadu

Before applying sentiment analysis to our extracted reviews, we use various text pre-processing steps to find valuable features for our classification model. Most of the task is accomplished using the Natural Language Tool Kit library along with VADER. We analyze the various steps of text processing to see how it changes our data and if the changes add value to the data.

For training and testing the model, PyTorch is used, which is a machine learning library specially designed for applications involving natural language processing and computer vision. It has in-built support for deep neural networks- our main model for the analyzer. Python also has support for libraries like Pandas, NLTK, Scikit-learn which all are used for performing basic functions required by the model. This as a whole, enables quick and efficient categorization of data to keep both companies and customers satisfied.

\section{LITERATURE SURVEY}

[1] Studies inferred that twitter is platform for vast data generation consisting of different cultures. Data was extracted using python library (Tweepy) and further cleansed using NLP toolkit. A corpus was used to understand the polarity (good vs bad) of the tweets. A list for dictionary was generated to materialize the data. Another dictionary was used to create associated hashtags. Hashtag value is generated to make a co-occurrence network which would visualize the data. Concluding the paper, unpopular tags saw higher count as compared to popular and expected tags in positive and negative aspects. The study helps to infer that the main focus of the company does not necessarily define its central talking point. Companies may need a leaner approach and this sentiment analysis highlights that fact.

Content creation by public is increasing at a high rate. These contents consist of opinion about the companies and their products. These feedback not just depict the user's mindset but also influence the mindset of others. [2] For systematic understanding on the meaning of feedback, we have three aspects: linguistic resource, sentiment classification and opinion summarization. Linguistic resource and sentiment classification attempt to classify the lingual into positive and negative feedback using various approaches such as PMI and conjunction method. Opinion summarization focuses on giving an overall opinion considering all polarities. [3] The paper accounts for the customer feedback of the food commodities. The sentiment analysis could be done in these ways: documents, sentence and aspect level with document and sentence level eventually being the same. 
The customer feedback is taken in the form of points ranging from 1 to 5 with points being +3 as positive otherwise negative. The results portrayed in the histogram define the rating of the food. The study however does not account for the hidden sentiments. The result is depicted using word cloud with colour of word defined their polarity, I.e. good -> yellow(bright), not -> black (dark, negative). It is established that [4] current paper is low on the depth of database and that hidden reviews are in massive in number whose polarity can help in the actual analysis. The n-grams also play a vital role in defining the sentiment. The study goes on the state that higher n-gram improves and fuels the negative polarity comments. The performance of the classifier is also not sensitive towards number of features. Also filtering the sentences does not guarantee better performance instead reduces a measure by $1.9 \%$. Mostly the study done earlier on consumer review or feedback are based on sentiment analysis by determining the polarity of the text. [5] In this study the word is converted to vector and algorithms are applied on them. This model also doesn't take into account n-gram model, but continuous bag of words (CBOW). It is also proved here that CBOW is more accurate as compared to normal sentiment analysis. Even though vector representation performs better spelling error accounting increases accuracy. [6] The author shows the possibility of achieving high accuracy classification of customer feedback using large feature vectors along with feature reduction based on log likelihood ratio and training a linear SVM. Feature Reduction is used for optimizing the performance of a linear classifier. [7]. Vader (Valence Aware Dictionary for sentiment Reasoning) is a library designed for sentiment analysis of social media text. The rate and volume of content along with contextual sparseness and abbreviated sentences are the major challenges faced by the author. VADER is considered to be better than human raters and also well understands the context of the text compared to benchmarks used by the author. Naïve Bayes classification when compare to SVMs perform poorly. [8] Machine learning techniques prove to be more efficient than human's manual work. The features indicating on-topic sentences are considered to be the important next step for identification of features. [9] Features favorable for sentiment analysis are Bag of Words Model, Syntactic and Semantic properties to capture the relations among words, handling negations. Supervised classification algorithms like Maximum Entropy, Naive Bayes, Decision Trees and unsupervised classification algorithms like K-means clustering available in NLTK Platform is used for classification purposes based on subjectivity and polarity. The authors use text data and build models for classifying text into positive, negative and neutral sentiment. The models used by the author are unigram, tree kernel-based and feature-based model. [10] The kernel-based model outperforms the other two models by a large margin. The author also shows that the text-specific features make the classification better.

\section{METHODOLODY}

This project works in two parts, i.e. scraping webpages and performing sentimental analysis, further categorizing these reviews into broad categories for analysis. It follows an elimination approach, by removing the techniques which don't provide good enough efficiency.

\section{A. Web Scarping and Sentiment Analysis}

The customer visits the website and searches for the product he desires. Then he writes a feedback for it which is helpful for other customers and for technical teams to get information on sales techniques to be followed later.

The methodology is proposed as follows:

- Firstly, these reviews are pre-processed in order to remove cases, punctuation, stop words, common words and perform tokenization and lemmatization for effective splitting of text. It is followed by feature extraction to get a more information out of the text.

- $\quad$ Then, these reviews are extracted, along with the ratings from first page of website and stored in separate lists since URL for the page is unique, Similarly, other pages are requested and data is extracted. This data gets stored in a 2D list where each list contains reviews \& corresponding ratings from different pages.

- $\quad$ This data is placed from 2D list to 1D list containing all the reviews in one list and their corresponding rating in a different list. Then merging the review, rating list of first page and other pages, a data frame is created.

- This data frame is used for Sentiment Analysis of reviews by applying the concepts of Natural Language Processing (NLP), by training the classifier and working on getting maximum efficiency. 82\% accuracy was achieved using this model.

\section{B. Categorization of Segmented Reviews}

Now that, the extracted data is classified as Positive, Negative and Neutral, we decided to further classify these into broad categories such as Product defective, Promo code issue, Size not available etc. to help the sellers get a better insight into what the needs of the customers are.

sellers get a better insight into what the needs of the customers are.

The methodology followed is proposed as follows:

- Each category of segmented comments is taken separately and worked on. For the case of negative comments, the goal of the project is building a system which will group similar complaints together and provide trends over time over these groups.

- The model follows two main approaches: Unsupervised and Supervised- Unsupervised approach uses a simple n-gram model to calculate the similarity between sentences using cosine similarity which is a part of Sklearn toolkit. A predicted score is calculated which is evaluated to measure efficiency. This model could not accurately classify the comments so we decided to eliminate this approach.

- We then tried the supervised approach and used a recurrent neural network (RNN) which makes sure the word order is taken into consideration. This model is found to be faster than simple feed forward neural network. Passing out reviews through this model and training over it, achieved an accuracy of about $45 \%$ which is pretty high given our data set was random. 
Architecture for RNN is shown in Fig. 1.

- Now finally, to analyze trends over these, we used K-means clustering to divide our data into further broad categories. The results were compared for variety of date periods.

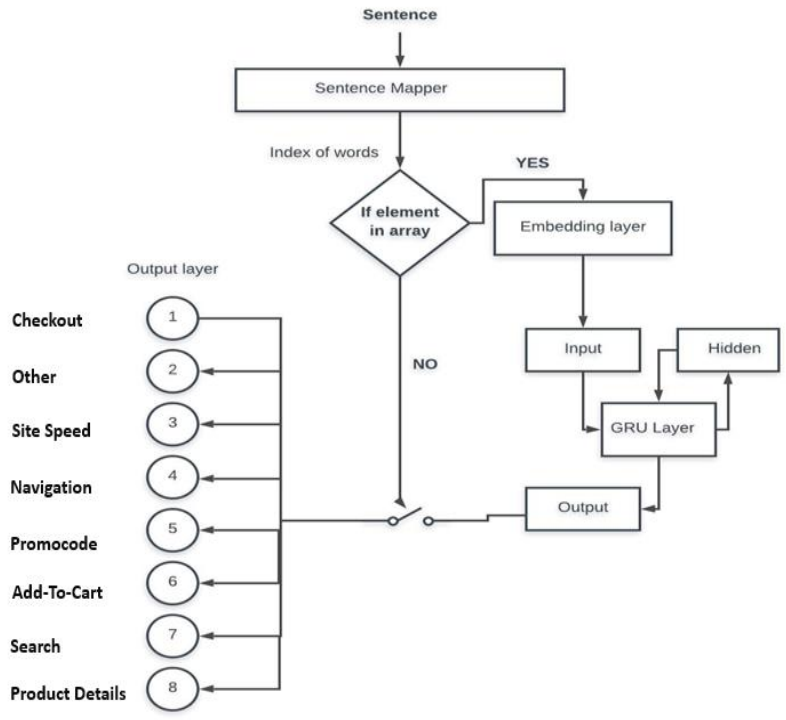

Fig.1. RNN architecture

\section{IMPLEMENTATION}

Fig.2. shows that model's accuracy was found to be $60 \%$. This means that our text classification model was not very efficient and needs changes in its feature set to better classify the tweets. Since the feature set in the previous model was not good enough, we decided to add new features to the data TF-IDF Columns. The model improved significantly as the model accuracy increased to $82 \%$ for sentiment analysis.

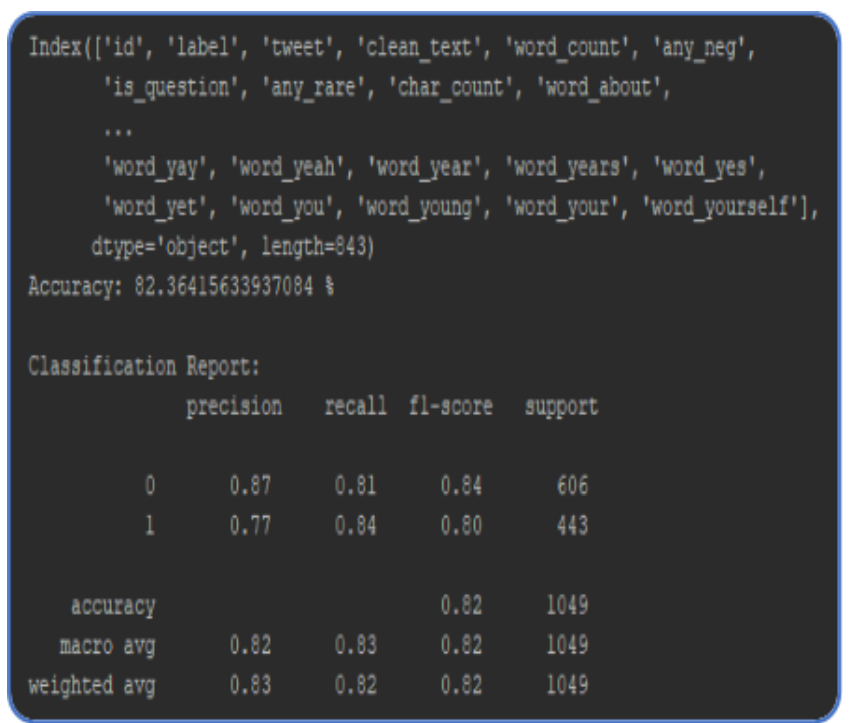

Fig.2. Classification Model Evaluation

Using Vader, we determined the sentiment score of each text. Vader returns four polarity scores - positive, negative, neutral \& compound. The following Fig displays the distribution of review sentiments among good reviews and bad reviews. Vader classifies the good reviews as positive with a higher compound score and bad reviews as negative having low compound score.

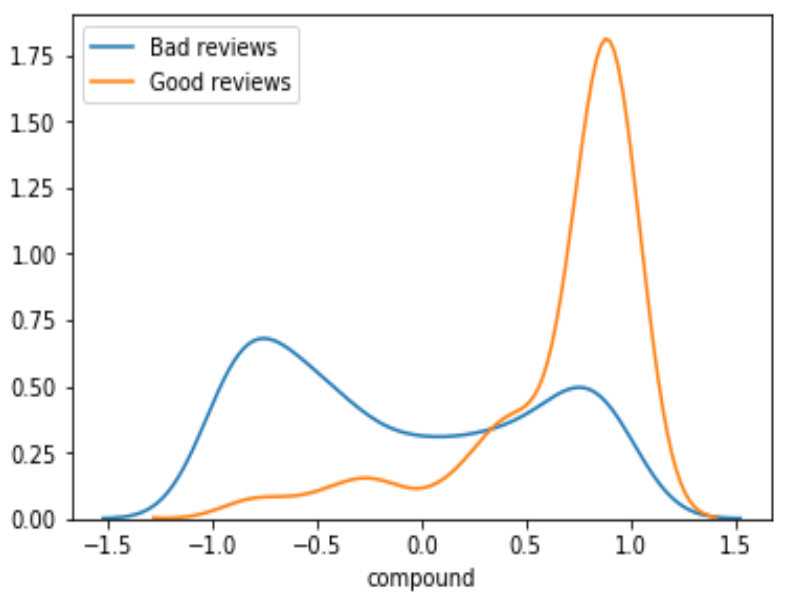

Fig.3. Sentiment Distribution

Fig4. Shows the eliminated unsupervised model which gave us an accuracy of about 36\%. It has constant readings for almost all the categories which doesn't give us much scope for improvement. Fig. 5. shows an accuracy of $45 \%$ for the supervised model, which is much better.

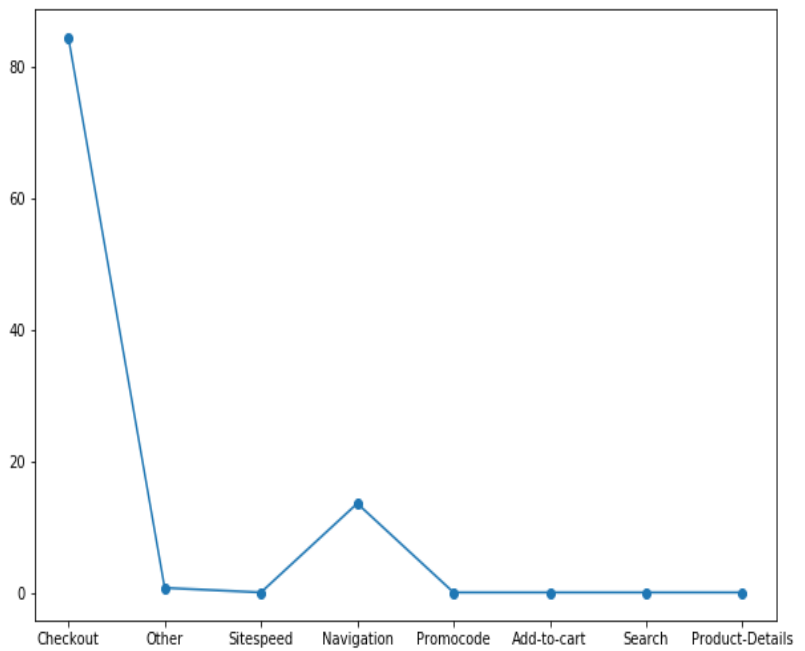

Fig. 4. Unsupervised model

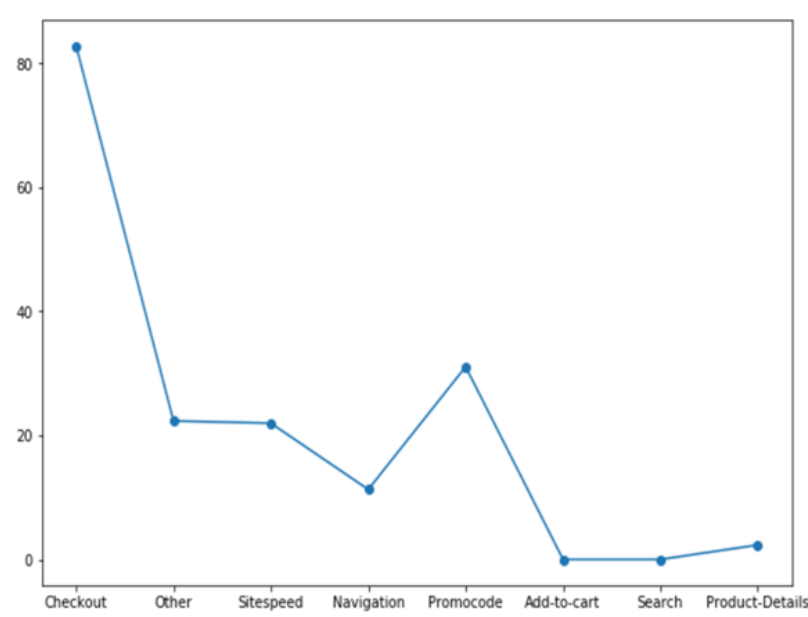

Fig. 5. Supervised model 
In Fig.6. the sentence "s1" is passed through the RNN Model, after training we observe that our model successfully predicts the label same as the actual label.

I have been attempting to checkout with my super cash and wes unable to do so My code is pQl.6FiV7XX4Q from my enail Predicted label:Pronocods

Actual libeli,Prombode

\section{Fig.6. Expected output}

\section{CONCLUSION}

After training and passing the model through the network, we were successfully able to categorize the reviews into sub-categories. We achieved a good accuracy and can use this model to automatically categorize the reviews as soon as they are published online, i.e., make it a self-learning model. This drastically reduces the work of employees from around 10 days for about 10000 reviews to less than 30 seconds for each review. Further, we can enable chat-bot support directly to the website, so that the customer can directly publish the comment to be chat-bot and it will notify the corresponding team directly by applying the model internally within itself. Customer Feedback Analyzer eases our work and can be incorporated into day-to-day operations to increasing efficiency and save time.

\section{REFERENCES}

1. M. Gamon, "Sentiment classification on customer feedback data," Proceedings of the 20th international conference on Computational Linguistics - COLING 04, 2004.

2. D. Lee, O.-R. Jeong, and S.-G. Lee, "Opinion mining of customer feedback data on the web," Proceedings of the 2nd international conference on Ubiquitous information management and communication - ICUIMC 08, 2008.

3. S. P and L. Mary, "Sentiment analysis of online food review using customer rating.," International Journal of Pure and Applied Mathematics.

4. H. Cui, V. Mittal, and M. Datar, "Comparative experiments on Sentiment classification for Online Product Reviews."

5. B. Bansal and S. Srivastava, "Sentiment classification of online consumer reviews using word vector representations," Procedia Computer Science, vol. 132, pp. 1147-1153, 2018.

6. Gamon, M. Sentiment classification on customer feedback data. Proceedings of the 20th international conference on Computational Linguistics - COLING 04 (2004). doi:10.3115/1220355.1220476

7. Hutto, C. and Gilbert, E. (n.d.). [online] Comp.social.gatech.edu. Available http://comp.social.gatech.edu/papers/icwsm14.vader.hutto.pdf

8. Pang, B., Lee, L. \&Vaithyanathan, S. Thumbs up? Proceedings of the ACL-02 conference on Empirical methods in natural language processing - EMNLP 02 (2002). doi:10.3115/1118693.1118704

9. Yessenov, K. and Misailovi c, S. (2009). [online] People.csail mitedu. Available http://people.csail.mit.edu/kuat/courses/6.863/report.pdf

10. Agarwal, A., Xie, B., Vovsha, I. \& Rambo, O. Sentiment Analysis with Twitter Data. SentimentAnalysis of Twitter Data (2018). doi:10.4135/9781526468857

\section{AUTHORS PROFILE}

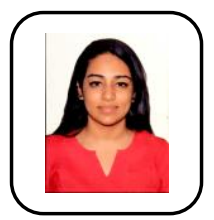

Sanya Taneja is currently pursuing B.Tech in Computer Science from Vellore Institute of Technology, Vellore. She has worked on projects like Spot-A-SpotEfficient car parking system, Vocabulary Enhancer which published in Springer journal. She has done various technical internships and worked on projects involving latest technologies like deep learning, natural

language processing, blockchain.

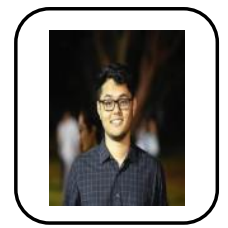

Kartikeya Jha is persuading his Bachelor of Technology degree in Computer Science from Vellore Institute of Technology, Vellore of batch 2020. His current research interest is in Natural language processing and artificial intelligence. He has worked on various projects related to software development and full stack development. He has also interned in various technical fields in esteemed technical

companies.

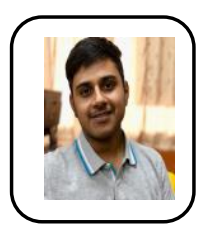

Nakul Lakhotia is currently pursuing his B.Tech in Computer Science from Vellore Institute of Technology, Vellore. His research areas include Machine Learning, Deep Learning, Natural Language Processing, and Computer Vision. He has done various internships and worked on projects like Text Classification, Chatbots and Sentiment Analysis.

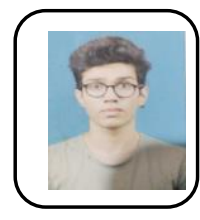

Vedanta Kapoor is currently pursuing his B.Tech Degree in Computer Science from VIT Vellore. He has worked on various project like automated home, sentiment analysis, and AI chat bots. He has done various technical internships. He is good at android coding and competitive coding and has a good knowledge of database management. He enthusiastic about data science and financial engineering.

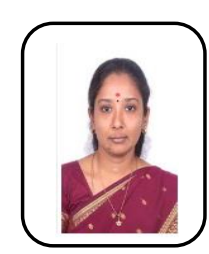

Swarnalatha Purushotham is an Associate Professor, in the School of Computer Science and Engineering, VIT University, at Vellore, India. She pursued her Ph.D. degree in Image Processing and Intelligent Systems. She has published more than 75 papers in International Journals/International Conference Proceedings/National Conferences. She is having $18+$ years of teaching experiences. She is a professional member of IACSIT, CSI, ACM, IACSIT, IEEE (WIE), ACEEE. She is an Editorial board member/reviewer of International/ National Journals and Conferences. Her current research interest includes Image Processing, Remote Sensing, Artificial Intelligence, Bigdata Analytics, Deep Learning and Software Engineering. 